\title{
Feature integration across the lifespan: stickier stimulus-response bindings in children and older adults
}

\author{
Bernhard Hommel $^{1}$ *, Jutta Kray ${ }^{2}$ and UIman Lindenberger ${ }^{2,3}$ \\ ${ }^{1}$ Leiden Institute for Psychological Research and Leiden Institute for Brain and Cognition, Netherlands \\ ${ }^{2}$ Department of Psychology, Saarland University, Saarbrücken, Germany \\ ${ }^{3}$ Center for Lifespan Psychology, Max Planck Institute for Human Development, Berlin, Germany
}

\section{Edited by:}

Wilfried Kunde,

Julius-Maximilians-Universitaet

Wuerzburg, Germany

Reviewed by:

Matthew J. C. Crump, Vanderbilt

University, USA

Daryl Wilson, Queen's University,

Canada

\section{${ }^{*}$ Correspondence:}

Bernhard Hommel, Cognitive

Psychology Unit, Institute for

Psychological Research, Leiden

University, Wassenaarseweg 52,

2333 AK Leiden, Netherlands.

e-mail: hommel@fsw.leidenuniv.nl
Humans integrate the features of perceived events and of action plans into episodic event files. Here we investigated whether children (9-10 years), younger adults (20-31 years), and older adults (64-76 years) differ in the flexibility of managing (updating) event files. Relative to young adults, performance in children and older adults was more hampered by partial mismatches between present and previous stimulus-response relations, suggesting less efficient updating of episodic stimulus-response representations in childhood and old age. Results are discussed in relation to changes in cortical neurochemistry during maturation and senescence.

Keywords: attention, feature binding, integration, aging, development

\section{INTRODUCTION}

The human brain encodes, processes, and stores the different features of events in a distributed fashion (e.g., DeYoe and Van Essen, 1988). This raises the question of how it keeps track of all the neural codes involved and how it integrates the features belonging to the same event. It has been argued that integration processes create bindings between the codes of the features involved and thereby form something like an object file (Kahneman et al., 1992). Numerous findings suggest that object files indeed exist. Particularly suggestive is what Kahneman et al. have called the "reviewing effect," which occurs in tasks where multifeatured prime and probe stimuli are presented. Performance is usually good if all features of a task-irrelevant prime stimulus are repeated on the probe or if all features change, but it is impaired if some features are repeated while others change (Kahneman et al., 1992; Hommel, 1998; for an overview, see Hommel, 2004). Apparently, registering the prime stimulus is sufficient to bind its feature codes together, so that reviewing one or more of its features retrieves the whole binding. If this binding matches the present feature combination (which is the case with complete repetition), no particular problem arises; but if one or more feature codes mismatch (as in the case of partial repetitions), code conflict is created, resulting in errors, temporal delays, or both (Hommel, 2004). Interestingly, partial-repetition costs of this sort are not only found for relations between visual features but for relations between visual features and responses as well. That is, if people respond to sequences of stimuli, they perform worse if a (task-related) stimulus feature is repeated while the response changes, and vice versa (Hommel, 1998). This suggests that the codes of stimulus and response features are integrated into "event files" in each given trial, which are retrieved in the next trial if at least one element is repeated (Hommel, 2004).

The aim of this study was to investigate possible changes in the handling of feature bindings across the lifespan by comparing children, younger adults, and older adults. Maturation and senescence represent natural manipulations of brain systems and brain-behavior relations (e.g., Lindenberger et al., 2006) that can be suspected to play a role in the cognitive handling of event files. Note that partial-repetition costs can only be observed if two conditions are met: feature codes need to be integrated when encountering a given stimulus-response episode (the prime event) and this created binding needs to be retrieved when encountering a subsequent stimulus-response episode (the probe event). Interestingly, studies that attempted to dissociate these two types of processes suggest that the binding process proper is more or less automatic: among other things, the degree and strength of binding two given features is independent from the frequency and probability of their co-occurrence (Hommel and Colzato, 2009; Colzato et al., 2006a) and the availability of attentional resources (Hommel, 2005).

In contrast, the retrieval process has been shown to be sensitive to task instructions and individual differences, which suggests a certain degree of control. For example, bindings involving task-relevant features have a stronger impact on behavior; this suggests that they are more likely to be retrieved (Hommel, 1998, 2007), presumably because preparing for a task involves increasing the gain of codes from task-relevant feature dimensions (Hommel, 2004, 2010). Moreover, partial-repetition costs have been found to be more - rather than less - pronounced in populations that are unlikely to possess particularly well-functioning binding processes, such as individuals with low fluid intelligence (Colzato 
et al., 2006b). Given that executive-control functions are related to fluid intelligence (Duncan et al., 2000), this suggests that people differ with respect to the efficiency of managing episodic representations, presumably in the degree to which they are able to inhibit bindings from previous encounters if necessary and/or to update these bindings to match with the present feature combination (Colzato et al., 2006b). This interpretation also fits with the demonstration that neurofeedback that increases neural gammaband activity over the frontal cortex leads to both improvements in memory retrieval and more selectivity in retrieving event files (Keizer et al., 2010a,b). These findings suggest that, if anything, cognitive development and aging might affect the retrieval and/or updating of event files and the control of this retrieval/updating process in particular. Indeed, fluid intelligence - which reduces partial-repetition costs (Colzato et al., 2006b) - and cognitive control are assumed to be closely related (e.g., Duncan et al., 2000). Moreover, both fluid intelligence and the control of the intentional retrieval of episodic long-term memories (Lepage et al., 2000; Buckner, 2003) have been associated with the frontal cortex, a neural structure that is among the last to mature in late childhood and adolescence (Lenroot and Giedd, 2006) and among the first to show marked signs of senescence in later adulthood and old age (Raz et al., 2005, 2008). Along these lines, we expected that partialrepetition costs decrease from childhood to early adulthood and increase from early to late adulthood. In other words, younger adults should be better in handling event files than children and older adults.

If age does have an impact on event-file management, it would be interesting to see which feature relations are affected. Recent studies have revealed that different kinds of relations are affected by different environmental stimulations. For example, the spontaneous binding of different visual features (e.g., shape and color) is sensitive to the administration of muscarinic-cholinergic agonists and antagonists, whereas stimulus-response bindings (e.g., shape and a particular key press) are entirely unaffected (Colzato et al., 2004, 2005). Likewise, partial-overlap costs associated with the (un)binding of visual features correlate with each other but do not correlate with the costs associated with stimulus-response (un)binding (Colzato et al., 2006c). This suggests that different neurotransmitter systems are involved in integrating features into objects on the one hand and in object-action coupling on the other. Indeed, stimulus-response integration (or, more precisely, the integration of the task-relevant stimulus feature and the response) has been shown to be selectively targeted by manipulations that are likely to affect dopaminergic pathways, such as the presentation of affect-inducing pictures (Colzato et al., 2007a) and the generation of stress (Colzato et al., 2008). Along the same lines, individual eye blink rate - an indicator of dopaminergic functioning - correlates with the strength of stimulus-response integration but not with effects related to the integration of other features (Colzato et al., 2007b). Interestingly, intelligence has also a selective effect on the integration of the task-relevant stimulus feature and the response (Colzato et al., 2006b). One may speculate that this is because fluid intelligence and stimulus-response integration processes are related to the same, presumably dopaminergic neurotransmitter system (Previc, 1999). If we further consider that the efficiency of dopaminergic neuromodulation has been related to both age and fluid intelligence (Bäckman et al., 2006), it would make sense to expect that the integration of task-relevant stimulus features (shape in the present study) and the response is particularly sensitive to age.

The task we used was adopted from Hommel (1998), see Figure 1. Participants responded manually to two successive visual stimuli (S1 and S2) that varied in shape and location. Whereas the second response (R2) was signaled by the shape of S2, the first response (R1) was precued in advance of S1 (which allowed the independent manipulation of $\mathrm{S} 1$ and $\mathrm{R} 1$ ) and carried out to the mere onset of $\mathrm{S}^{1}$. In this design, interactions between shape repetition and location repetition (showing worse performance on S2/R2 if one feature repeats but the other alternates) can be taken to reflect the aftereffect of visual integration, while interactions between shape repetition and response repetition, and between location repetition and response repetition, reflect aftereffects of visuomotor integration. We were thus interested to see whether age would modulate interactions between stimulusand response-repetition effects on R2 and expected that such a modulation would be particularly pronounced for the interaction between shape and response repetition - the two task-relevant (stimulus/response feature) dimensions.

\footnotetext{
${ }^{1}$ One might argue that this design could create a conflict produced by S1/R1 processing. As S2 and R2 are associated through the instructed stimulus-response rules, a partial repetition of the relevant stimulus feature and the response implies that S2/R2 were preceded by a combination that violates these rules (e.g., if the letter X as S2 requires a right-hand keypress, the combination of an X as S1 and a left-hand keypress implies a rule violation). However, previous studies suggest that this factor does not play a role. For instance, $\mathrm{R} 1$ reaction times are not affected by rule violations (Hommel, 1998) and interactions between stimulus and response repetitions can be observed in free-choice tasks, where no stimulus-response rules are applied (Hommel, 2007). Given that children often have problems with free-choice tasks, we opted for a forced-choice design in the present study.
}

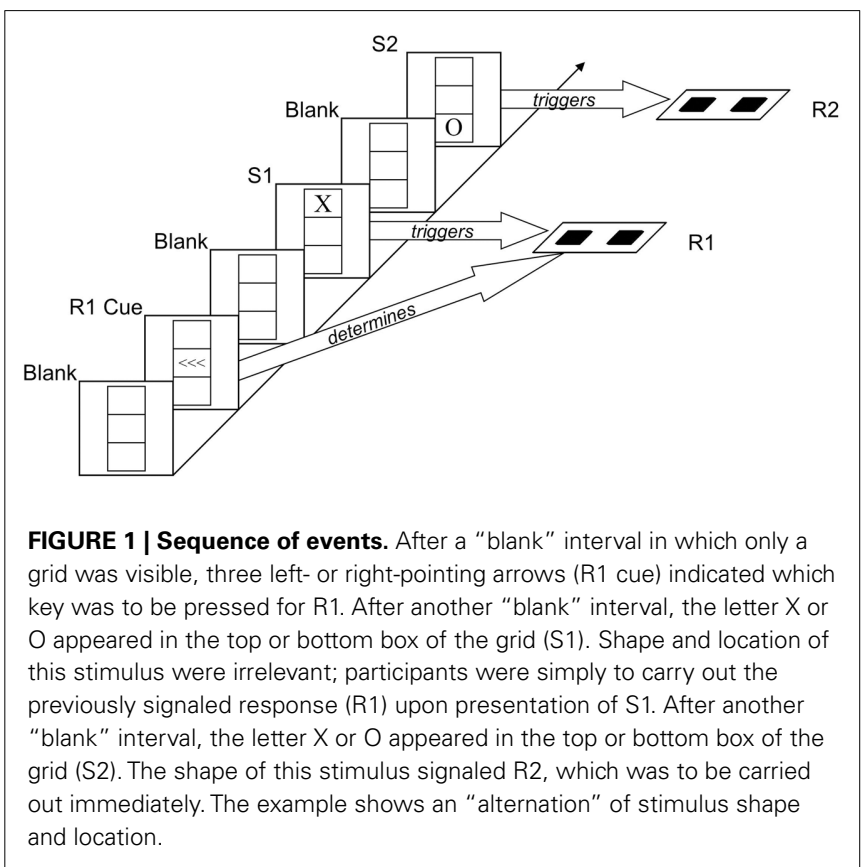




\section{MATERIALS AND METHODS PARTICIPANTS}

Twenty-two children (9-10 years, 11 female), 22 younger adults (20-31 years, 14 female), and 21 older adults (64-76 years, 11 female) took part for pay. All reported having normal or correctedto-normal vision.

\section{APPARATUS AND STIMULI}

The experiment was controlled by a PC attached to a color monitor. Participants faced a grid made of three gray square outlines, vertically arranged, as illustrated in Figure 1. From viewing distance of about $60 \mathrm{~cm}$, each of these frames measured $2.6^{\circ} \times 3.1^{\circ}$. The green letters $\mathrm{O}$ and $\mathrm{X}$, taken from the standard text font, served as S1 and S2 alternatives, which were presented in the top or bottom frame. Response cues appeared in the middle frame, with rows of three left- or right-pointing arrow heads, indicating a left and right keypress, respectively. Responses were made by pressing the left or right shift-key of the computer-keyboard with the corresponding index finger.

\section{PROCEDURE AND DESIGN}

Participants carried out two responses per trial. R1 was a simple reaction with the left or right key, as indicated by the response cue. It was to be carried out as soon as S1 appeared, independent of its shape or location. Participants were informed that there was no systematic relationship between S1 and R1, or between S1 and S2, and they were encouraged to respond to the mere onset of S1 only, disregarding the stimulus' attributes. R2 was a binary-choice reaction to the shape of S2; half of the participants carried out left and right keypresses to the letters $\mathrm{O}$ and $\mathrm{X}$, respectively, while the other half received the opposite mapping. The sequence of events in each trial is shown in Figure 1. Next to the intertrial of $3 \mathrm{~s}$ a response cue signaled R1 for $3 \mathrm{~s}$, followed by a 1-s interval in which only the gray grid remained on the screen. Then, $\mathrm{S} 1$ appeared for $600 \mathrm{~ms}$ or until R1 was given, followed by a further grid-only interval of $800 \mathrm{~ms}$. If R1 was incorrect or not given within $1 \mathrm{~s}$, the trial started again from the beginning. Then, S2 appeared to signal R2, that is, the stimulus-onset asynchrony between S1 and S2 was 1,400 ms. S2 stayed until R2 was given or $2 \mathrm{~s}$ had passed. Each session comprised 96 trials, composed by three repetitions of each of the 32 factorial combination of the two shapes ( $\mathrm{O}$ and $\mathrm{X}$ ) and locations (top vs. bottom) of S2, and the repetition or alternation of stimulus shape and location, and the response. Errors were signaled by a beep and error trials were repeated at a random position in the remainder of the session.

\section{RESULTS}

In all statistical analyses, alpha level was set to $p<0.05$. $F$ and $p$ values, together with partial eta square $\left(\eta^{2}\right)$ wherever appropriate, are reported for significant ANOVA results, $p$ values are provided for non-significant findings of theoretical relevance. Theoretically driven follow-up comparisons were carried out by means of $t$-test, $p$ values are provided for significant contrasts and for non-significant findings of theoretical relevance.

We also compared the sizes of partial-repetition costs (effect sizes). Note that these costs are diagnosed by two-way interactions, that is feature $\mathrm{X}$ (e.g., shape) and $\mathrm{Y}$ (e.g., response location) are assumed to be integrated if the effects of repeating (vs. alternating) feature $\mathrm{X}$ and repeating (vs. alternating) feature $\mathrm{Y}$ interact. Computing the size of such a two-way interaction requires the consideration of four data points - the interaction term. We therefore calculated the partial-repetition cost for a given interaction between factors $\mathrm{X}$ and $\mathrm{Y}$ as the difference between the reaction time (RT)/error mean for partial repetitions (feature $\mathrm{X}$ repeated and feature $\mathrm{Y}$ alternated, or vice versa) and the RT/error mean for complete repetitions and "complete" alternations. For instance, the partial-repetition RT cost related to the shape-byresponse interaction in a given individual or age group would be $\mathrm{PRC}_{\text {shape,response }}=\left(\mathrm{RT} 2_{\text {shape repeated,response alternated }}+\mathrm{RT} 2_{\text {shape }}\right.$ alternated,response repeated $) / 2-(\mathrm{RT} 2$ shape repeated,response repeated $+\mathrm{RT} 2$ shape alternated,response alternated $) / 2$. Note that partial-repetition costs are immune to possible, but theoretically less relevant, main effects of feature repetition; a value close to zero means that the repetition effects of the two given features do not interact; a value greater than zero indicates a "binding-aftereffect-type" interaction of the sort discussed in this article.

\section{R1}

Children, younger adults, and older adults carried out R1 in 449, 394 , and $479 \mathrm{~ms}$, respectively, and the corresponding error rates were $1.9,0.5$, and $1.3 \%$. Both RTs and error rates varied with Age, $F(2,62)=5.47, p>0.01, \eta^{2}=0.15$, and $F(2,62)=4.72, p<0.05$, $\eta^{2}=0.13$, respectively. Pairwise comparisons showed that younger adults were faster, $p<0.05$, and more accurate, $p<0.005$, than children and older adults, $p<0.005$ and $p<0.05$, whereas children and older adults did not differ in response speed, $p>0.3$, or accuracy, $p>0.3$. Anticipatory responses ( RT $<150 \mathrm{~ms}$ ) were very rare $(<0.02 \%)$ and did not differ between the three age groups, $p>0.3$. Response omissions did vary with Age, $F(2,62)=7.35, p<0.001$, $\eta^{2}=0.19$, due to fewer omissions in young adults $(1.0 \%)$ than in children (5.0\%), $p<0.001$, and older adults $(3.2 \%), p<0.005$, whereas children and old adults did not differ, $p>0.15$.

\section{R2}

Children, younger adults, and older adults omitted the response in $0.5,0$, and $0.1 \%$ of the trials, respectively, and the corresponding age effect failed to reach significance, $p>0.08$. From the remaining data, mean RTs and proportions of errors (PEs) were calculated and analyzed as a function of Age group and the repetition vs. alternation of the Shape and Location of the stimulus $(\mathrm{S} 1 \rightarrow \mathrm{S} 2)$, and the repetition vs. alternation of the Response $(\mathrm{R} 1 \rightarrow \mathrm{R} 2)$, see Table 1 for means. Four-way ANOVAs were performed by treating Age group as between-participants factor and the three stimulus or response relations as within-participants factors.

\section{Reaction times}

The outcome of the RT analysis falls into three categories. First, the analysis yielded main effects of Shape repetition, $F(1,62)=29.02, p<0.001, \eta^{2}=0.32$, Response repetition, $F(1,62)=4.78, p<0.05, \eta^{2}=0.07$, and Age, $F(2,62)=3.97$, $p<0.05, \eta^{2}=0.11$, indicating that performance was better with shape repetitions than shape alternations, with response repetitions than response alternations, and in young adults than in children and older adults, which did not differ significantly, 
Table 1 | Means of mean reaction times for responses (R2) to stimulus 2 (RT; in ms) and percentages of errors on R2 (PE), as a function of age group, the repetition (vs. alternation) of responses and the stimulus features shape and location.

Age group

\begin{tabular}{|c|c|c|c|c|c|c|c|c|c|c|c|}
\hline \multicolumn{4}{|c|}{ Children } & \multicolumn{4}{|c|}{ Young adults } & \multicolumn{4}{|c|}{ Old adults } \\
\hline \multicolumn{12}{|c|}{ Response } \\
\hline \multicolumn{2}{|c|}{ Repeated } & \multicolumn{2}{|c|}{ Alternated } & \multicolumn{2}{|c|}{ Repeated } & \multicolumn{2}{|c|}{ Alternated } & \multicolumn{2}{|c|}{ Repeated } & \multicolumn{2}{|c|}{ Alternated } \\
\hline RT & PE & RT & PE & $\mathbf{R T}$ & PE & $\mathbf{R T}$ & PE & RT & PE & $\mathbf{R T}$ & PE \\
\hline 693 & 24.1 & 595 & 4.4 & 557 & 7.6 & 488 & 0.6 & 695 & 1.8 & 583 & 0.7 \\
\hline 599 & 14.0 & 606 & 14.8 & 515 & 2.0 & 540 & 1.7 & 620 & 1.1 & 647 & 0.7 \\
\hline 672 & 19.7 & 646 & 14.1 & 540 & 7.4 & 518 & 1.7 & 683 & 3.9 & 614 & 0.4 \\
\hline 552 & 4.4 & 622 & 22.3 & 508 & 1.7 & 547 & 7.4 & 554 & 0.4 & 642 & 4.9 \\
\hline
\end{tabular}

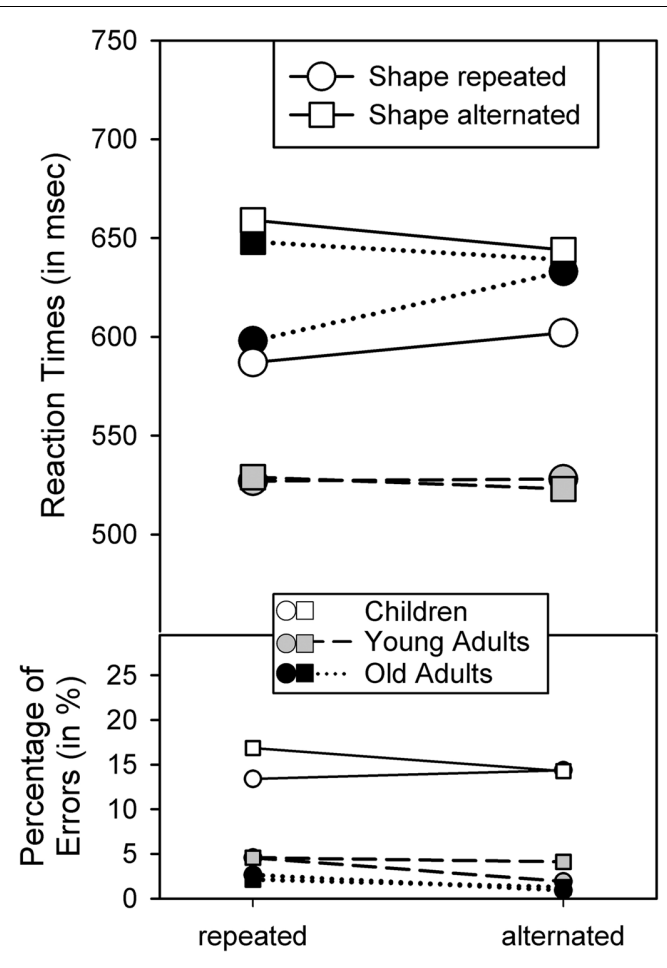

Stimulus location

FIGURE 2 | Reaction times and percentage of errors for R2, as a function of age group and the repetition vs. alternation of stimulus location and stimulus shape.

$p>0.8$. Age and Shape repetition were also involved in a two-way interaction, $F(2,62)=10.88, p<0.001, \eta^{2}=0.26$. As Figure 2 suggests, the shape-repetition benefit was reliable only in children, $F(1,21)=24.65, p<0.001$, and older adults, $F(1,20)=13.23$, $p<0.005$, but not in young adults, $p>0.8$.

Second, the main effects of Shape and Response repetition were qualified by two-way interactions between Shape and Location, $F(1,62)=10.52, p<0.005, \eta^{2}=0.14$, Shape and Response, $F(1,62)=119.65, p<0.001, \eta^{2}=0.66$, and Location and Response, $F(1,62)=40.10, p<0.001, \eta^{2}=0.39$. Figures 2 and 3 show that these interactions followed the common partialrepetition cost pattern with relatively good performance if both stimulus features, or a stimulus feature and the response, either repeat or alternate, while performance is relatively poor in conditions in which one factor repeats and the other alternates. Of course, this pattern could be further mediated by main effects, such as the shape-repetition RT benefit of stimulus repetition in children (see upper left panel in Figure 3), which in our case destroyed the standard cross-over interaction by moving the two shape-repeated data points downward. Nevertheless, if one considers these mediating factors, the underlying cross-over pattern is visible in all groups and for all feature combinations.

Third, and most importantly, the shape-by-response interaction was further qualified by Age group, $F(2,62)=4.27, p<0.05$, $\eta^{2}=0.12$. $t$-Tests (two-tailed) of the corresponding partialrepetition costs (i.e., the interaction terms, calculated as described above) revealed that this three-way interaction was due to that the shape-by-response effect was reliably more pronounced in older adults than in younger adults, $t(41)=3.19, p<0.005$, and tended to be more larger in older adults than in children, $p>0.07$, while children and young adults did not differ, $p>0.36$. Neither the interaction of shape and location repetition nor that of location and response repetition was reliably modified by age, $p>0.18$ and $p>0.13$, respectively. However, it is possible that this lack of three-way interactions with age was due to the fact that the underlying two-way interactions were less pronounced than the shape-by-response interaction in the first place. Indeed, when we analyzed the effect sizes from the three two-way interactions (shape/response, shape/location, and location/response; as described above) together as a function of age, there were only main effects of Age, $F(2,62)=4.60, p<0.05, \eta^{2}=0.13$, and of effect type, $F(2,124)=28.11, p<0.001, \eta^{2}=0.31$, indicating that the shape-by-response effect was stronger than the other two interactions. Interestingly, effect type did not interact with age, $p>0.2$, suggesting that age affected all three types of interactions similarly.

Inspecting Figures $\mathbf{2}$ and $\mathbf{3}$ indicates that more pronounced two-way interactions came along with higher RT levels. This raises 


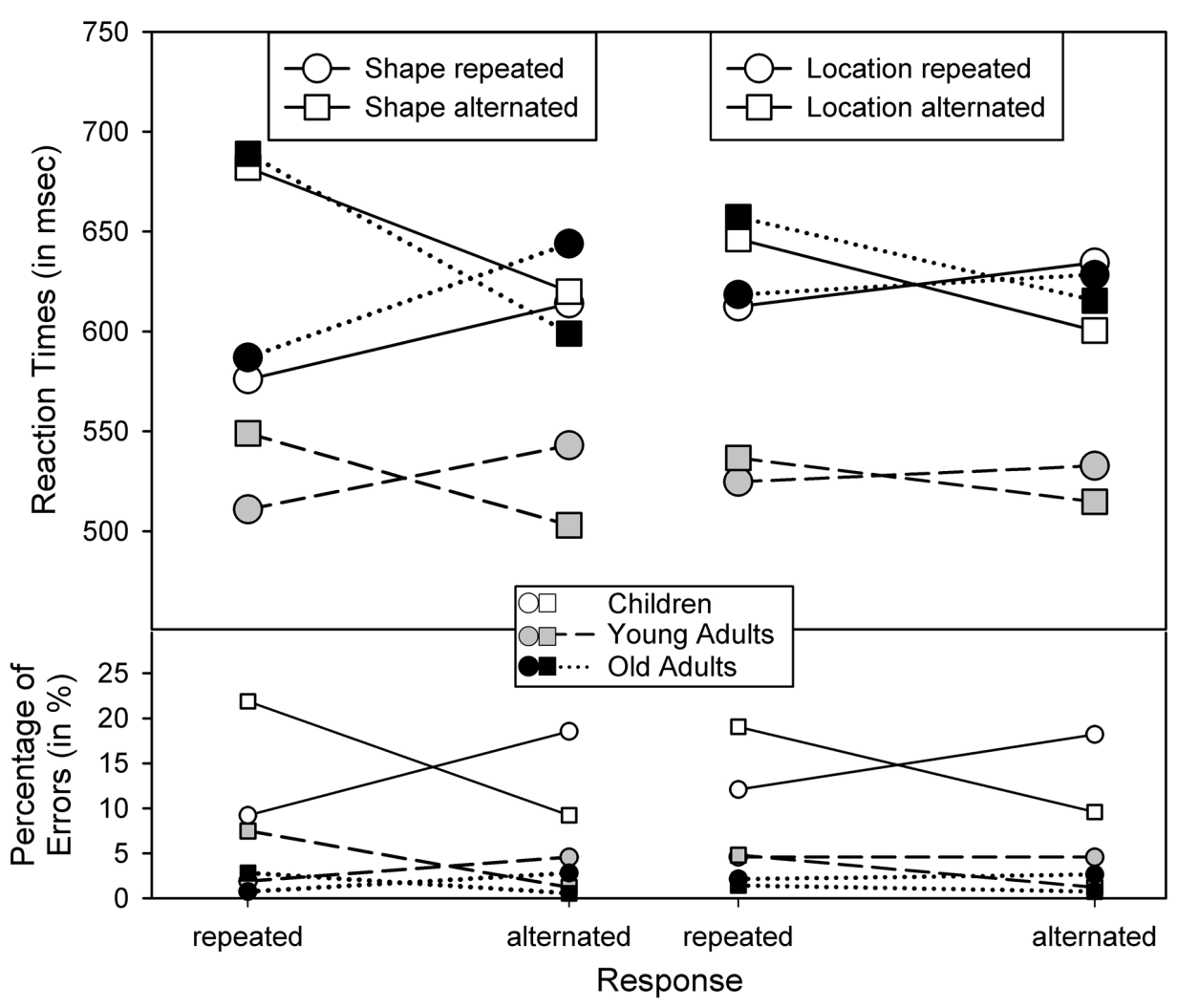

FIGURE 3 | Reaction times and percentage of errors for R2, as a function of age group and the repetition vs. alternation of stimulus shape and response (left panel) and the repetition vs. alternation of stimulus location and response (right panel).

the possibility that the RT level as such - or an underlying general factor, such as response speed (Salthouse, 1996) - "explains" the extent of the interactions. To test this possibility, we reanalyzed the shape-by-response effect (i.e., the corresponding partial-repetition costs ore interaction terms) in an ANOVA with Age group as factor and RT from the "easiest" condition (all stimulus/response features alternated) as an estimate of the general RT level as covariate. However, the age effect remained reliable, $F(2,61)=3.19, p<0.05, \eta^{2}=0.95$, suggesting that response speed may explain some, but certainly not all of this effect.

\section{Errors}

The error analysis yielded main effects of Age, $F(2,62)=83.18$, $p<0.001, \eta^{2}=0.73$, and Location, $F(1,62)=6.47, p<0.05$, $\eta^{2}=0.09$, due to higher error levels in children $(14.7 \%)$ than in younger adults $(3.8 \%)$ and older adults $(1.7 \%)$, and somewhat more errors to location repetitions $(7.4 \%)$ than to alternations $(6.1 \%)$ - the well-known inhibition of return (Posner and Cohen, 1984). The two-way interactions between Shape and Response, $F(1,62)=79.89, p<0.001, \eta^{2}=0.56$, and Location and Response, $F(1,62)=24.98, p<0.001, \eta^{2}=0.29$, were further modified by three-way interactions including Age, $F(2,62)=16.14, p<0.001$, $\eta^{2}=0.34$, and, $F(2,62)=10.73, p<0.001, \eta^{2}=0.26$, respectively. As Figure 3 shows, partial-repetitions yielded more errors than complete repetitions or alternations, and this interaction was particularly pronounced in children (younger and older adults did not differ, $p=0.08$ and $p=0.51)$. There was also a three-way interaction of Shape, Location, and Response, $F(1,62)=4.89, p<0.05, \eta^{2}=0.07$, indicating particularly good performance in the condition with all three features alternating - a rather common observation (see Hommel and Colzato, 2004).

To test whether the general accuracy level might "explain" the different sizes of the interaction effects in the age groups, we again (as for RTs) re-analyzed the two reliable interactions in ANOVAs with Age group as factor and the error rates from the "easiest" condition (all stimulus/response features alternated). As in RTs, the age effect remained reliable, which was true for both shape/response costs, $F(2,61)=16.38, p<0.001, \eta^{2}=0.35$, and location/response costs, $F(2,61)=8.13, p<0.001, \eta^{2}=0.21$. This suggests that general accuracy does not account for partial-overlap costs.

\section{DISCUSSION}

The aim of this study was to examine age differences in the efficiency to handle (i.e., inhibit and/or update) bindings of codes representing features of visual events and self-performed actions by assessing the aftereffects of processing one stimulusresponse episode on performance on another. We were able to replicate previous findings that repeating one member of a particular combination of stimulus features or of a stimulus and a response feature impairs performance if the other 
member changes: partial-repetition costs that is. This suggests that encountering a feature combination or a feature-response combination creates automatic bindings between the codes of the features involved - event files in the sense of Hommel (1998, 2004).

More importantly, the size of these partial-repetition costs differed across the age groups. As expected, aging effects were particularly pronounced with respect to the two task-relevant features shape (which signaled R2) and response location (which defined R2). This finding might indicate that binding and/or the retrieval of bindings is restricted to codes of task-relevant features. However, the overall data pattern suggests that it rather reflects the fact that interactions between shape and location, and between location and response, were somewhat less pronounced in the first place, so that the age factor might have been left with a smaller statistical operation space. More specifically, there might be two, presumably independent factors at work.

First, the size of interactions between stimulus- and responserepetition effects is obviously affected by task relevance, in the sense that task-relevant features are more likely to be involved in interactions than task-irrelevant features are (a well-known effect pattern; e.g., Hommel, 1998). This might be due to the attentional top-down modulation of feature coding when encoding and/or retrieving a stimulus-response episode (Hommel, 2004). Given that the relationship between effects related to task-relevant and irrelevant features did not vary as a function of age (i.e., age did not interact with effect type in the effect-size analysis), this topdown modulation is unlikely to be affected by development or aging.

Second, the impact that a retrieved event file has on ongoing processes, and/or the efficiency with which the resulting conflicts are resolved, seems to vary with age. However, this age-related modulation is more visible in the data the more pronounced the modulated effect is, which favors effects related to bindings involving task-relevant features. In that sense, the age-independent attentional factor might constrain the behavioral consequences of the age-dependent efficiency in handling bindings and the consequences of their retrieval without interacting with this process directly.

Another interesting observation is that how age modulates the impact of binding on performance seems to depend on the measure being considered: RTs were more sensitive to the effect of aging, showing a more pronounced effect in older adults than in younger adults or children. In contrast, the error rates were more sensitive to developmental changes from childhood to early adulthood, showing a more pronounced effect for children than for young and old adults. On the one hand, this might indicate that the processes underlying the worse performance in children and in older adults are different, so that the cognitive gains (from childhood to young adulthood) and losses (from young to old adulthood) have different underlying causes. On the other hand, however, older adults are known to be particularly accurate (Smith and Brewer, 1995), which might have compensated for possible error effects and have moved some effects from error rates to RTs. In any case, our findings suggest that RTs and error rates are not equally sensitive to binding-related effects in children and older adults.

Taken together, our observations suggest the existence of a kind of inverted U-shape function relating the efficiency of handling event files to age. What processes might be responsible for this function? As noted already, Colzato et al. (2006b) found that the efficiency in updating episodic representations is positively related to fluid intelligence. Tests of fluid intelligence, such as the Raven's used by Colzato and colleagues, typically require that individuals constantly reconfigure the material in their mind to induce the correct (i.e., most coherent and parsimonious) solution. Physiologically, this alternation of binding and partial un-binding/re-binding of event features has been assumed to require the locking and unlocking of partially overlapping neuronal groups and oscillatory states, processes assumed to critically depend on the dynamic coupling between dopaminergic neuromodulation and GABAergic activity (cf. Durstewitz et al., 1999). Given that the efficiency of dopaminergic neuromodulation is highly related to both age and fluid intelligence (Bäckman et al., 2006), age changes in dopaminergic neuromodulation may help to explain age differences in stimulus-response feature reconfiguration, or "un-binding/re-binding" efficiency (Schmiedek et al., 2009). As an outline for future research, we propose that the ability to flexibly reconfigure overlapping features of sensorimotor events is a fundamental determinant of lifespan changes in more complex forms of cognition.

Interestingly, inverted $U$-shape performance/age functions very similar to our present finding have been reported from studies on explicit feature binding in conjunction search (e.g., Trick and Enns, 1998; Hommel et al., 2004) and episodic-memory tasks (Chalfonte and Johnson, 1996; Li et al., 2005). However, there is also converging evidence that the increasing and the decreasing flanks of the function often do not mirror each other perfectly. In the present study, this was obvious from the fact that deficits in children affected RTs and error rates in different ways than they did in older adults - presumably reflecting the strategy of older adults to prefer accuracy over speed. Studies on explicit binding for episodic memory have revealed similar patterns. For instance, Cowan et al. (2006) obtained an inverted U-shape relating the recall of visual feature conjunctions to age, but under some conditions additional deficits were observed in the oldest participants. As the authors suggest, older adults may be more likely to rely on compensatory strategies like using familiarity cues, which depending on the task and the conditions may compensate for, or at least camouflage deficits in explicit binding. Even though more research on this issue is necessary, the employment of strategies that work for some but not other tasks may account for the rather inconsistent picture with regard to the impact of aging on explicit memory retrieval (e.g., Naveh-Benjamin et al., 2004; Parra et al., 2009).

\section{ACKNOWLEDGMENTS}

Support for this research by the Max Planck Institute for Human Development and by grants from Deutsche Forschungsgemeinschaft (DFG) to Bernhard Hommel (HO1430/8-2) and Jutta Kray (KR1884/3-2) is gratefully acknowledged. 


\section{REFERENCES}

Bäckman, L., Nyberg, L., Lindenberger, U., Li, S. C., and Farde, L. (2006). The correlative triad among aging, dopamine, and cognition: current status and future projects. Neurosci. Biobehav. Rev. 30, 791-807.

Buckner, R. L. (2003). Functionalanatomic correlates of control processes in memory. J. Neurosci. 23, 3999-4004.

Chalfonte, B. L., and Johnson, M. K. (1996). Feature memory and binding in young and older adults. Mem. Cognit. 24, 403-416.

Colzato, L. S., Erasmus, V., and Hommel, B. (2004). Moderate alcohol consumption in humans impairs feature binding in visual perception. Neurosci. Lett. 360, 103-105.

Colzato, L. S., Fagioli, S., Erasmus, V., and Hommel, B. (2005). Caffeine, but not nicotine enhances visual feature binding. Eur. J. Neurosci. 21, 591-595.

Colzato, L. S., Kool, W., and Hommel, B. (2008). Stress modulation of visuomotor binding. Neuropsychologia 46, 1542-1548.

Colzato, L. S., van Wouwe, N. C., and Hommel, B. (2007a). Feature binding and affect: emotional modulation of visuo-motor integration. Neuropsychologia 45, 440-446.

Colzato, L. S., van Wouwe, N. C., and Hommel, B. (2007b). Spontaneous eyeblink rate predicts the strength of visuomotor binding. Neuropsychologia 45, 2387-2392.

Colzato, L. S., Raffone, A., and Hommel, B. (2006a). What do we learn from binding features? Evidence for multilevel feature integration. $J$. Exp. Psychol. Hum. Percep. Perf. 32, 705-716.

Colzato, L. S., van Wouwe, N. C., Lavender, T. J., and Hommel, B. (2006b). Intelligence and cognitive flexibility: fluid intelligence correlates with feature "unbinding" across perception and action. Psychon. Bull. Rev. 13, 1043-1048.

Colzato, L. S., Warrens, M. J., and Hommel, B. (2006c). Priming and binding in and across perception and action: a correlational analysis of the internal structure of event files. Q. J. Exp. Psychol. 59, 1785-1804.
Cowan, N., Naveh-Benjamin, M., Kilb, A., and Saults, J. S. (2006). Lifespan development of visual working memory: when is feature binding difficult? Dev. Psychol. 42, 1089-1102.

DeYoe, E. A., and Van Essen, D. C. (1988). Concurrent processing streams in monkey visual cortex. Trends Neurosci. 11, 219-226.

Duncan, J., Seitz, R. J., Kolodny, J., Bor, D., Herzog, H., Ahmed, A., Newell, F. N., and Emslie, H. (2000). A neural basis for general intelligence. Science 289, 457-460.

Durstewitz, D., Kelc, M., and Güntürkün, O. (1999). A neurocomputational theory of the dopaminergic modulation of working memory functions. J. Neurosci. 19, 2807-2822.

Hommel, B. (1998). Event files: evidence for automatic integration of stimulus response episodes. Vis. Cogn. 5, 183-216.

Hommel, B. (2004). Event files: feature binding in and across perception and action. Trends Cogn. Sci. 8, 494-500.

Hommel, B. (2005). How much attention does an event file need? J. Exp. Psychol. Hum. Percep. Perf. 31, 1067-1082.

Hommel, B. (2007). Feature integration across perception and action: event files affect response choice. Psychol. Res. 71, 42-63.

Hommel, B. (2010). “Grounding attention in action control: the intentional control of selection," in Effortless Attention: A New Perspective in the Cognitive Science of Attention and Action, ed. B. J. Bruya (Cambridge, MA: MIT Press), 121-140.

Hommel, B., and Colzato, L. S. (2004). Visual attention and the temporal dynamics of feature integration. Vis. Cogn. 11, 483-521.

Hommel, B., and Colzato, L. S. (2009). When an object is more than a binding of its features: evidence for two mechanisms of visual feature integration. Vis. Cogn., 17, 120-140.

Hommel, B., Li, Z. H., and Li, S.C. (2004). Visual search across the life span. Dev. Psychol. 40, 545-558.

Kahneman, D., Treisman, A., and Gibbs, B. J. (1992). The reviewing of object files: object-specific integration of information. Cogn. Psychol. 24, 175-219.

Keizer, A. W., Verment, R., and Hommel, B. (2010a). Enhancing cognitive control through neurofeedback: a role of gamma-band activity in managing episodic retrieval. Neuroimage 49, 3404-3413.

Keizer, A. W., Verschoor, M., Verment, R., and Hommel, B. (2010b). The effect of gamma enhancing neurofeedback on measures of featurebinding flexibility and intelligence. Int. J. Psychophysiol. 75, 25-32.

Lenroot, R. K., and Giedd, J. N. (2006). Brain development in children and adolescents: insights from anatomical magnetic resonance imaging. Neurosci. Biobehav. Rev. 30, 718-729.

Lepage, M., Ghaffar, O., Nyberg, L., and Tulving, E. (2000). Prefrontal cortex and episodic memory retrieval mode. Proc. Natl. Acad. Sci. U.S.A. 97, 506-511.

Li, S.-C., Naveh-Benjamin, M., and Lindenberger, U. (2005). Aging neuromodulation impairs associative binding: a neurocomputational account. Psychol. Sci. 16, 445-450.

Lindenberger, U., Li, S.-C., and Bäckman, L. (eds). (2006). Methodological and conceptual advances in the study of brain-behavior dynamics: a multivariate lifespan perspective. Neurosci. Biobehav. Rev. 30, 713-892. [Special issue].

Naveh-Benjamin, M., Guez, J., Kilb A., and andReedy, S. (2004). The associative memory deficitof older adults: further support using facename associations. Psychol. Aging 19, 541-546.

Parra, M. A., Abrahams, S., Logie, R. H., and Della Sala, S. (2009). Age and binding within-dimension features in visual short-term memory. Neurosci. Lett. 449, 1-5.

Posner, M. I., and Cohen, Y. (1984). "Components of visual orienting," in Attention and Performance X: Control of Language Processes, eds $\mathrm{H}$. Bouma and D. G. Bouwhuis (Hillsdale, NJ: Erlbaum), 531-556.

Previc, F. H. (1999). Dopamine and the origins of human intelligence. Brain Cogn. 41, 299-350.

Raz, N., Lindenberger, U., Ghisletta, P., Rodrigue, K. M., Kennedy, K. M., and Acker, J. D. (2008).
Neuroanatomical correlates of fluid intelligence in healthy adults and persons with vascular risk factors. Cereb. Cortex 18, 718-726.

Raz, N., Lindenberger, U., Rodrigue, K. M., Kennedy, K. M., Head, D., Williamson, A., Dahle, C. Gerstorf, D., and Acker, J. D. (2005). Regional brain changes in aging healthy adults: general trends, individual differences and modifiers. Cereb. Cortex 15, 1676-1689.

Salthouse, T. A. (1996). The processingspeed theory of adult age differencesin cognition. Psychol. Rev. 103, 403-428.

Schmiedek, F., Li, S.-C., and Lindenberger, U. (2009). Interference and facilitation in spatial working memory: age-associated differences in lure effects in the n-back paradigm. Psychol. Aging 24, 203-210.

Smith, G. L., and Brewer, N. (1995). Slowness and age: Speed-accuracy mechanisms. Psychol. Aging 10, 238-247.

Trick, L., and Enns, J. (1998). Lifespan changes in attention: the visual search task. Cogn. Dev. 13, 369-386.

Conflict of Interest Statement: The authors declare that the research was conducted in the absence of any commercial or financial relationships that could be construed as a potential conflict of interest.

Received: 21 February 2010; paper pending published: 23 March 2010; accepted: 27 September 2011; published online: 01 November 2011.

Citation: Hommel B, Kray J and Lindenberger $U$ (2011) Feature integration across the lifespan: stickier stimulusresponse bindings in children and older adults. Front. Psychology 2:268. doi: 10.3389/fpsyg.2011.00268

This article was submitted to Frontiers in Cognition, a specialty of Frontiers in Psychology.

Copyright (C) 2011 Hommel, Kray and Lindenberger. This is an open-access article subject to a non-exclusive license between the authors and Frontiers Media $S A$, which permits use, distribution and reproduction in other forums, provided the original authors and source are credited and other Frontiers conditions are complied with. 